\title{
The Pharmacokinetics and Metabolism of Neomangiferin, a Major Bioactive Component in Anemarrhena asphodeloides
}

Yu Gao\#, Huihui Liu\#, Pei Hu, Mingcang Chen, Xiaoting Tian, Zhixiong Li* and Chenggang Huang*

"Authors contributed equally

Shanghai Institute of Materia Medica, Chinese Academy of Sciences, Shanghai, China

\begin{abstract}
As a natural derivative of bioactive xanthone mangiferin, neomangiferin exhibits a wide spectrum of bioactivities. However, studies have yet to elucidate the metabolic pathways and pharmacokinetics of orally administered neomangiferin in vivo. In this study, a simple and accurate high-performance liquid chromatography/diode array detector method was developed and validated to simultaneously determine neomangiferin and its active metabolite mangiferin in plasma after oral and intravenous administration of neomangiferin to rats. Chromatographic separation was performed in a C18 column by using water containing $0.1 \%$ formic acid-acetonitrile $(88: 12, \mathrm{v} / \mathrm{v})$ at a flow rate of $0.7 \mathrm{~mL} / \mathrm{min}$. The calibration curves of neomangiferin and mangiferin in rat plasma were linear at $150-15000(r=0.9999)$ and $50-1500 \mathrm{ng} / \mathrm{mL}(r=0.9994)$, respectively. The relative standard deviations of intraday and inter-day precision were below $10.7 \%$, and accuracy ranged from 94.0 to $105.5 \%$ for both analytes. Experimental results showed that intravenously and orally administered neomangiferin was partly metabolized into mangiferin. $T_{\text {max }}$ of neomangiferin and mangiferin was low $(0.05 \mathrm{~h})$, and the two components were eliminated at $\mathrm{t}_{12}$ of 0.95 and $0.73 \mathrm{~h}$, respectively. Similar to that of mangiferin, the oral bioavailability of neomangiferin was also low (about $0.32 \%$ ). Thirty-three metabolites were detected and absolutely or tentatively identified on the basis of mass spectral fragmentation pattern and elution order or were confirmed by using available reference standards. Our findings suggested that neomangiferin undergoes rapid and extensive phases I and II metabolic processes, such as deglycosylation, dehydroxylation, methylation, glycosylation, glucuronidation, and sulfation, in rats. In general, the pharmacokinetic and metabolic profiles, including poor bioavailability, high absorption and elimination rates, extensive biotransformation, of neomangiferin are similar to those of mangiferin to some degrees.
\end{abstract}

Keywords: Neomangiferin; Mangiferin; Pharmacokinetics; Metabolism; HPLC-DAD

\section{Introduction}

As a derivative of bioactive xanthone mangiferin, neomangiferin (Figure 1) is a natural xanthone C-glycoside mainly obtained from the well-known traditional Chinese medicine rhizoma of Anemarrhena asphodeloides [1]. This plant is officially listed in the Chinese Pharmacopeia under the name Zhimu. Various biological activities have been discovered in neomangiferin. For instance, neomangiferin exhibits antidiabetic activity in KK-Ay mice by decreasing insulin resistance and increasing insulin sensitivity $[2,3]$. Neomangiferin presents antiosteoporotic action in ovariectomized rats [4]. It also elicits anti-colitic effects by suppressing IL-17 and ROR $\gamma \mathrm{t}$ expression and inducing IL-10 and forkhead box P3 expression [5].

Neomangiferin and mangiferin co-exist, and they are considered the major active constituents in Zhimu [6]. Mangiferin has been investigated extensively because of its remarkable pharmacological effects, including antioxidant [7,8], anti-inflammatory [9], antidiabetic [10], anticancer [11], antibacterial [12], anti-HIV [13], and radioprotective activities [14]. Notably, neomangiferin is likely biotransformed to mangiferin in vivo [7].

With advanced knowledge of the pharmacological effects of neomangiferin and mangiferin, it is necessary to gain insights into the pharmacokinetic profiles in vivo because pharmacokinetics plays an increasingly crucial role in drug discovery and development processes; pharmacokinetics not only further supports toxicity or clinical trials but also optimizes drug candidates [15]. The metabolism and pharmacokinetic profiles of mangiferin have recently been examined [16-18]. Pharmacokinetic studies have indicated that mangiferin exhibits poor oral bioavailability $(1.2 \%)$ when orally administered with the isolated compound directly [19]. However, very few studies have been published on the pharmacokinetics and metabolism of neomangiferin as another major bioactive substance and structurally similar to mangiferin. Ma et al. [6] bioanalyzed neomangiferin after Rhizoma Anemarrhenae decoction is orally administered. Qiu et al. [20] developed an ultra-performance liquid chromatography (UPLC)-tandem mass spectrometry (MS/MS) method to simultaneously determine mangiferin and neomangiferin in rat plasma after co-administration of mangiferin and neomangiferin. However, the pharmacokinetics of orally administered neomangiferin and its active metabolite-mangiferin has yet to be reported. The biotransformation of neomangiferin in vivo also remains unknown.

In this study, a simple and accurate method was established to determine neomangiferin and its metabolite mangiferin in rat plasma simultaneously. The proposed method was successfully applied to analyze neomangiferin pharmacokinetically after it was orally and intravenously administered to rats. The metabolites of neomangiferin in rats systemically and comprehensively investigated through LCESI-IT-MS $^{\mathrm{n}}$ to establish possible metabolic pathways. Our findings helped elucidate the therapeutic effects and mechanism of action of neomangiferin, and promote its further development.

*Corresponding authors: Chenggang Huang, Shanghai Institute of Materia Medica, Chinese Academy of Sciences, Shanghai 201203, China, Tel: +862120231963; E-mail: cghsimm@126.com

Zhixiong Li, Shanghai Institute of Materia Medica, Chinese Academy of Sciences, Shanghai 201203, China, Tel: +862120231963; E-mail: zxli0715@126.com

Received May 23, 2016; Accepted June 06, 2016; Published June 10, 2016

Citation: Gao Y, Liu H, Hu P, Chen M, Tian X, et al. (2016) The Pharmacokinetics and Metabolism of Neomangiferin, a Major Bioactive Component in Anemarrhena asphodeloides. Med chem (Los Angeles) 6: 385-394. doi:10.4172/21610444.1000374

Copyright: (c) 2016 Gao Y, et al. This is an open-access article distributed under the terms of the Creative Commons Attribution License, which permits unrestricted use, distribution, and reproduction in any medium, provided the original author and source are credited. 


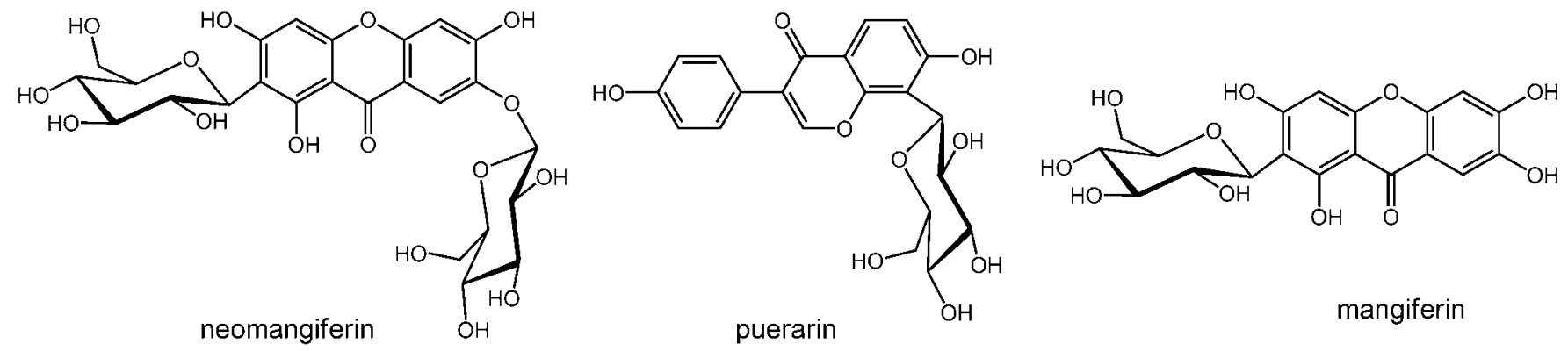

Figure 1: Structures of neomangiferin, puerarin (internal standard), and mangiferin.

\section{Materials and Reagents}

\section{Chemicals and reagents}

Neomangiferin (98\% purity) for dosing was purchased from Tianjin Zhongxin Pharmaceutical Group Corporation (Tianjin, China). Puerarin (for internal standard), neomangiferin, and mangiferin (for reference) were purchased from the National Institute for Control of Pharmaceutical and Biological Products (Beijing, China). Streptozotocin, streptomycin, and neomycin sulfate were purchased from Sigma-Aldrich (St. Louis, MO). Acetonitrile and formic acid used for HPLC (HPLC grade) were purchased from Fisher Chemical Co., Inc. (CA, USA). All other analytical grade reagents were from Sinopharm Chemical Reagent Co., Ltd. (Shanghai, P. R. China). Triple deionized water was prepared in a Milli-Q system (Millipore, Billerica, MA, USA) in our laboratory whenever necessary. Waters Oasis HLB cartridges $\left(60 \mathrm{mg}, 3 \mathrm{~cm}^{3}\right)$ for sample pretreatment were purchased from Waters (Milford, MA).

\section{Animals}

Male Wistar rats $(250 \pm 10 \mathrm{~g})$ were purchased from Shanghai SLAC Laboratory Animal Co. (Shanghai, China). All animals were housed in an environmentally controlled room and acclimated for one week before use.

For preparation of pseudo-germ-free animals, neomycin sulfate $(100 \mathrm{mg} / \mathrm{kg})$ and streptomycin $(100 \mathrm{mg} / \mathrm{kg})$ were orally administered to 10 conventional rats twice per day for 6 days. The experiments were performed 2 days after the final administration.

For metabolism and pharmacokinetic study, animals were divided into three drug-treated groups (ten healthy rats in group A after oral administration; ten healthy rats in group $\mathrm{B}$ after intravenous injection; ten pseudo-germfree rats in group $\mathrm{C}$ after oral administration; with five rats per group for urine and feces collecting and the rest for blood withdrawn) and one control group with six rats. All animals were fasted overnight with free access to water prior to a single oral dose (400 $\mathrm{mg} / \mathrm{kg}$ ) or intravenous injection $(1.8 \mathrm{mg} / \mathrm{kg})$. All animal studies were conducted according to protocols approved by the Review Committee of Animal Care and Use at the Shanghai Institute of MateriaMedica (Shanghai, China).

\section{Instrumentation and analytical conditions}

Quantification of neomangiferin and mangiferin in plasma: determination of neomangiferin and mangiferin in rat plasma was performed on an Agilent 1260 system (Agilent, Waldbronn, Germany) equipped with a quaternary pump, a photodiode array detector (DAD) working in the range of 190-400 nm, autosampler and a column compartment. The chromatographic separation was performed on an Inertsil ODS-3 column $(150 \mathrm{~mm} \times 4.6 \mathrm{~mm}, 5 \mu \mathrm{m}$, GL Sciences,
Tokyo, Japan) protected with a guard column $(10.0 \mathrm{~mm} \times 4.6 \mathrm{~mm}, 5$ $\mu \mathrm{m}$, Dikma Technologies, Beijing, China) eluted at $0.7 \mathrm{~mL} / \mathrm{min}$ with a mobile phase of acetonitrile-water containing $0.1 \%$ formic acid (12:88, $\mathrm{v} / \mathrm{v})$. The wavelength was set at $320 \mathrm{~nm}$ and the column temperature was kept at $25^{\circ} \mathrm{C}$. The injection volume was $20 \mu \mathrm{L}$. The chromatographic data were recorded and processed with Agilent ChemStation software.

Identification of neomangiferin metabolites in rat biological samples: separation was achieved on a reversed phase Inertsil ODS3 column $(4.6 \mathrm{~mm} \times 250 \mathrm{~mm}, 5 \mu \mathrm{m}$, GL Sciences, Tokyo, Japan $)$ connected to a Diamonsil C18 guard column $(2.1 \mathrm{~mm} \times 12.5 \mathrm{~mm}, 5$ $\mathrm{m}$, Dikma Technologies, Beijing, China). The column temperature was set at $25^{\circ} \mathrm{C}$ and the flow rate maintained at $0.8 \mathrm{~mL} / \mathrm{min}$. Mobile phase consisting of acetonitrile (A) and water containing $0.5 \%$ formic acid (B) used a linearly gradient program as follows: $10-23 \% \mathrm{~A}$ at $0-15 \mathrm{~min}$, hold for $5 \mathrm{~min}$, linearly gradient to $45 \% \mathrm{~A}$ in $25 \mathrm{~min}$, then linearly gradient to $61 \% \mathrm{~A}$ at $60 \mathrm{~min}$. An aliquot of $20 \mu \mathrm{L}$ of the purified sample was injected automatically into the HPLC system for LC-ESI-IT-MS ${ }^{\mathrm{n}}$ analysis. MS analyses were conducted in negative ion mode and the operating parameters were optimized as follows: collision gas, ultrahigh-purity helium (He); nebulizing gas, high-purity nitrogen $\left(\mathrm{N}_{2}\right)$; capillary voltage, $3.5 \mathrm{kV}$; end plate offset, $500 \mathrm{~V}$; nebulizer, 30 psi; drying gas flow rate, $10 \mathrm{~L} / \mathrm{min}$; drying gas temperature, $350^{\circ} \mathrm{C}$. For full scan MS analysis, the spectra were recorded in the range of 100-1000 Da.

\section{Preparation of stock and working solutions}

The standard stock solutions of neomangiferin, mangiferin, and puerarin were individually prepared in $50 \%$ methanol at $120 \mu \mathrm{g} / \mathrm{mL}$ and serially diluted to make the working solutions with $50 \%$ methanol. All stock and working solutions were stored at $-20^{\circ} \mathrm{C}$ until use.

\section{Preparation of calibration standards and quality control samples}

Calibration standards samples were prepared by spiking appropriate amount of the standard solutions in blank plasma $(200 \mu \mathrm{L})$ to yield final concentrations of $150,300,600,1200,3000,6000,15000 \mathrm{ng} / \mathrm{mL}$ for neomangiferin; 50, 100, 300, 600, 900, 1200, and $1500 \mathrm{ng} / \mathrm{mL}$ for mangiferin and $3000 \mathrm{ng} / \mathrm{mL}$ for puerarin. Three concentrations of quality control (QC) samples were prepared containing neomangiferin $(300,6000$, and $12000 \mathrm{ng} / \mathrm{mL})$, mangiferin $(100,600$, and $1200 \mathrm{ng} / \mathrm{mL})$, and puerarin $(3000 \mathrm{ng} / \mathrm{mL})$ in the same manner.

\section{Plasma sample preparation}

The plasma $(200 \mu \mathrm{L}$, containing the IS at the concentration of $3000 \mathrm{ng} / \mathrm{mL}$ ) was pretreated by protein precipitation with 3 volumes of acetonitrile-acetic acid (v/v, 9:1). The supernatant was separated and dried under nitrogen gas at $37^{\circ} \mathrm{C}$ after vortexing for $2 \mathrm{~min}$ and centrifuging at $15,000 \mathrm{~g}$ for $10 \mathrm{~min}$. The residues were reconstituted in $100 \mu \mathrm{L}$ of $50 \%$ methanol and centrifuged twice at $15,000 \mathrm{~g}$ for 10 
min. A $20 \mu \mathrm{L}$ aliquot was injected into the HPLC-DAD system for analysis.

\section{Method validation}

This assay was validated by validating specificity, lower limit of quantification (LLOQ), linearity, accuracy, precision, recovery, and stability.

Specificity was evaluated by analyzing blank plasma samples from six different sources to investigate the potential interferences for the analytes and IS. The linearity for each analyte (neomangiferin and mangiferin) was determined by plotting the peak area ratios of the analyte to the IS against the concentrations of neomangiferin and mangiferin in plasma in duplicate on five consecutive validation days.

The lowest limit of quantitation (LLOQ) of the assay was determined as the lowest concentration on the standard curve that could be reliably detected and reproducible with a precision equal to or less than $20 \%$ and accuracy between $80 \%$ and $120 \%$.

The precision and accuracy of the method were assessed by replicate analysis ( $n=5)$ of QC samples on the same day (intra-day) and also on five consecutive days (inter-day). The intra- and inter-day precisions were required to be below $15 \%$, and the accuracy within $\pm 15 \%$.

The extraction recovery of neomangiferin, mangiferin and IS (puerarin) was determined by comparing peak areas obtained after whole extraction of QC samples at three concentration levels for five replicates with those obtained from direct injections of standard solutions.

The stability of neomangiferin and mangiferin in rat plasma was assessed by analyzing three replicates of QC samples $(n=3)$ at three concentrations. The stability of $24 \mathrm{~h}$ was performed by the QC samples during analysis at room temperature. Long-term stability was tested after storage of samples at $-80^{\circ} \mathrm{C}$ for 25 days. The freeze/thaw stability was examined at three freeze/thaw cycles between $-80^{\circ} \mathrm{C}$ and room temperature. Samples were considered stable with the deviation from the nominal concentration within $\pm 15 \%$.

\section{Pharmacokinetic study}

The validated HPLC-DAD method was applied to simultaneously determine the plasma concentrations of neomangiferin and mangiferin in healthy rats after oral administration and intravenous injection of neomangiferin. Blood samples were withdrawn via the angular vein at $20 \mathrm{~min}, 40 \mathrm{~min}, 70 \mathrm{~min}, 2 \mathrm{~h}, 3 \mathrm{~h}, 4 \mathrm{~h}, 5 \mathrm{~h}, 6.5 \mathrm{~h}, 8 \mathrm{~h}, 9.5 \mathrm{~h}, 11 \mathrm{~h}$, and $24 \mathrm{~h}$ after oral administration and $3,6,10,20,30,45,60,90,120$, and $180 \mathrm{~min}$ after intravenous injection. Plasma was separated from blood placed in heparinized Eppendorf tubes after centrifuging at $4000 \times \mathrm{g}$ for $10 \mathrm{~min}$. All plasma samples were stored at $-80^{\circ} \mathrm{C}$ until analysis. Pharmacokinetic analysis was performed with WinNonlin software (Pharsight 6.2; NC, USA) utilizing non-compartmental analysis.

\section{Metabolism study of neomangiferin}

Urine and feces samples within $0-48 \mathrm{~h}$ post-treatment were collected and admixed separately. Plasma samples at each time point were combined for metabolic study after pharmacokinetic study. Each sample of urine $(400 \mu \mathrm{L})$ and plasma $(400 \mu \mathrm{L})$ was loaded onto a SPE column preconditioned with $3 \mathrm{~mL}$ of methanol and $3 \mathrm{~mL}$ of water subsequently. The cartridge was then washed with $3 \mathrm{~mL}$ of water and the analytes were eluted with $2 \mathrm{~mL}$ of methanol. Feces sample $(50 \mathrm{mg})$ was extracted by ultrasonication three times with $1 \mathrm{~mL}$ of methanol for $30 \mathrm{~min}$ each time. Drug containing plasma was precipitated with 3 volumes of acetonitrile: acetic acid (9:1). The supernatant was separated after votexing and centrifuging. Extracting solutions with different pretreatment methods described above were all dried under nitrogen gas over a water bath of $37^{\circ} \mathrm{C}$. The residues were reconstituted in 100 $\mu \mathrm{L}$ of methanol and centrifuged twice at $10,000 \times \mathrm{g}$ for $10 \mathrm{~min}$ prior to analysis. All the blank samples as control were prepared with the same method as drug containing samples.

\section{Results and Discussion}

\section{Liquid chromatography}

The representative chromatograms obtained from blank plasma, blank plasma spiked with the analytes and IS, and plasma sample after administration are shown in Figure 2. Neither endogenous interference nor metabolites appeared at the retention time of the analytes and IS. The retention time of neomangiferin, IS (puerarin) and mangiferin were $7.5,18.5$ and $20.7 \mathrm{~min}$, respectively.

\section{Method validation}

The calibration curves of neomangiferin and mangiferin in rat plasma were linear over the ranges $150-15000$ and 50-1500 ng/ $\mathrm{mL}$, respectively. The regression equations were $\mathrm{y}=0.0005 \mathrm{x}-0.0234$ $(r=0.9999)$ for neomangiferin and $y=0.0009 x+0.0115(r=0.9994)$ for mangiferin. The lowest limit of Quantitation (LLOQ) of neomangiferin and mangiferin in rat plasma were 150 and $50 \mathrm{ng} / \mathrm{mL}$, respectively.

The estimates of the intra- and inter-day precision and accuracy of the assay are presented in Table 1. For all the QC samples spiked with analytes at three concentration levels in rat plasma, the relative standard deviations (RSD) for both intra-day and inter-day precision were below $10.7 \%$, and the accuracy was within the range of $94.0 \%$ $105.5 \%$ for both analytes.

The extraction recoveries were $56.6 \% \pm 2.3 \%, 61.2 \% \pm 3.2 \%, 59.8 \%$ $\pm 1.9 \%$ for neomangiferin and $77.8 \% \pm 5.1 \%, 81.5 \% \pm 1.7 \%, 75.0 \% \pm$ $2.5 \%$ for mangiferin at three concentrations, respectively. Recovery for the internal standard was $75.8 \% \pm 2.9 \%$.

Table 2 suggests that the analytes were stable in plasma samples for $24 \mathrm{~h}$ at room temperature after preparation, within three freeze-thaw cycles, and after storage at $-80^{\circ} \mathrm{C}$ for 25 days.

\section{Pharmacokinetic study}

The concentration-time profiles of neomangiferin and its metabolite mangiferin in rat plasma following intravenous and oral neomangiferin administration are presented in Figure 3, and the selected pharmacokinetic parameters are summarized in Table 3.

After its intravenous administration, neomangiferin was partly metabolized into mangiferin rapidly and resulted in the increase of mangiferin in plasma, which was detected in the plasma from the first plasma sampling time ( $3 \mathrm{~min}$ ). Neomangiferin and mangiferin rapidly reached $\mathrm{T}_{\max }(0.05 \mathrm{~h})$. The $\mathrm{C}_{\max }$ of neomangiferin reached $10817.9 \mathrm{ng} /$ $\mathrm{mL}$, whereas the $\mathrm{C}_{\max }$ of the formed mangiferin reached $899.48 \mathrm{ng} / \mathrm{mL}$. Neomangiferin was eliminated at a $\mathrm{t}_{1 / 2}$ of $0.95 \mathrm{~h}$, whereas mangiferin was eliminated at $0.73 \mathrm{~h}$. The parent compound was detected in the plasma samples up to $180 \mathrm{~min}$, whereas the concentration of mangiferin was below the lowest limit of quantitation (LLOQ) after $90 \mathrm{~min}$. Compared with the pharmacokinetic parameters of neomangiferin and mangiferin in a previous publication [21], neomangiferin showed similar clearance after the sole intravenous administration of neomangiferin or the coadministration of neomangiferin and mangiferin. This finding indicates that the plasma clearance of neomangiferin may not be affected by the combinational administration of structurally similar xanthonoids. The area under the curve $\left(\mathrm{AUC}_{0-\infty}\right)$ of neomangiferin, calculated as 4578.82 
$\pm 680.4(\mathrm{ng} \mathrm{h}) / \mathrm{mL}$, was about 11.14 fold higher than that of mangiferin $(411.52 \pm 64.66(\mathrm{ng} \mathrm{h}) / \mathrm{mL})$.

When neomangiferin was orally administered to healthy rats, neomangiferin absorption and mangiferin formation were rapid; neomangiferin and mangiferin were detected in the plasma from the first plasma sampling time $(20 \mathrm{~min})$ and reached the maximum concentration shortly afterward $\left(\mathrm{T}_{\max } 0.67 \mathrm{~h}\right.$ for neomangiferin and $1.54 \mathrm{~h}$ for mangiferin). This finding is consistent with that in a previous

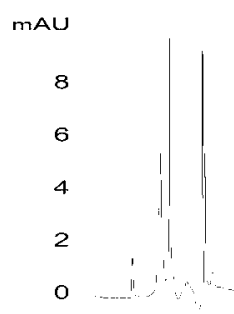

o

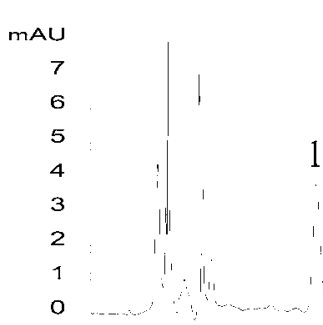

o

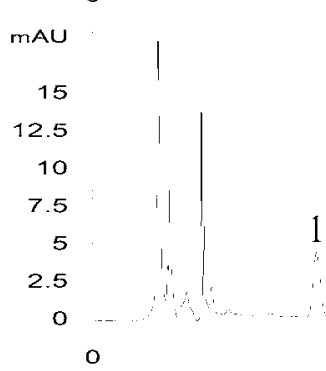

A

20 min

B

2

10

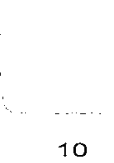

2

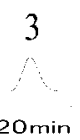

Figure 2: Chromatograms of neomangiferin (1), IS (2), and mangiferin (3) in Wistar rat plasma. (A) Blank plasma sample, (B) QC sample with $1200 \mathrm{mg} / \mathrm{mL}$ neomangiferin and $600 \mathrm{ng} / \mathrm{mL}$ mangiferin, and (C) plasma sample $1.17 \mathrm{~h}$ after neomangiferin was orally administered to rats. study in which neomangiferin was absorbed promptly at a $\mathrm{T}_{\max }$ of 0.58 $\mathrm{h}$ after oral administration of the Zi-Shen Pill extract [7]. The $\mathrm{C}_{\max }$ of neomangiferin reached $1804.28 \mathrm{ng} / \mathrm{mL}$, whereas the $t_{1 / 2}$ was only $0.95 \pm$ $0.31 \mathrm{~h}$, which is much lower than the corresponding value obtained after the oral administration of the Zi-Shen Pill [7]. The $\mathrm{C}_{\max }$ of mangiferin was $824.48 \mathrm{ng} / \mathrm{mL}$, and the plasma concentration slowly declined with a relatively long elimination $t_{1 / 2}$ of $3.22 \mathrm{~h}$. Hence, the elimination rate of mangiferin was slower than that of neomangiferin. Given the corresponding $\mathrm{AUC}_{0-\infty}$, the parent-to-metabolite exposure ratio was calculated to be about 1.12, which is lower than that of intravenous administration (11.14). This result indicates that a certain amount of neomangiferin could be transformed to its metabolite mangiferin in the gastrointestinal tract and subsequently absorbed in vivo. Notably, the $\mathrm{AUC}_{0-\infty}$ of the formed mangiferin in plasma was relatively high compared with that of the parent drug neomangiferin ( $\mathrm{AUC}_{\text {neomangiferin }}$ ! $\mathrm{AUC}_{\text {mangiferin }}=1.12$ ). Given the slow elimination rate of mangiferin, such AUC suggests that the active metabolite mangiferin appears to determine neomangiferin activity after oral administration. The oral bioavailability of neomangiferin (calculated using $\frac{\mathrm{AUC}(0 \rightarrow \mathrm{t}) \text { oral } \times 1.8}{\mathrm{AUC}(0 \rightarrow \mathrm{t}) \mathrm{i} \times \times 400} \times 100 \%$ ) was only $0.32 \%$, which is similar to that of mangiferin. These phenomena might be attributed to the low absorption and extensive metabolism of neomangiferin in vivo. Generally, neomangiferin exhibited rapid excretion and extremely low plasma concentration in the rats.

\section{Metabolic study of neomangiferin}

Neomangiferin-dosed biological samples were analyzed in parallel with corresponding blank controls using the developed LC-ESIIT-MS $^{\mathrm{n}}$ method. A total of 33 metabolites were detected and were further structurally identified. The extracted ion chromatograms of neomangiferin and its metabolites are presented in Figure 4. The identified compounds are summarized in Table 4.

Metabolites(M0, M5, M28, and M34) were unambiguouslyidentified as neomangiferin, mangiferin, norathyriol, and 1,7-dihydroxanthone, respectively, by comparing the characteristics of the fragmentation patterns and retention times with those of the standard compound. By contrast, the structures of 30 other metabolites (M1-M4, M6-M27, and M29-M33) were tentatively ascertained.

Metabolites M1 and M2 were eluted at 11.2 and $12.2 \mathrm{~min}$, respectively. Both of the compounds showed similar $[\mathrm{M}-\mathrm{H}]^{-}$ions at $m / z 597$, and their $\mathrm{MS}^{2}$ spectra yielded a major fragment at $\mathrm{m} / z 401$ because of the loss of a glucuronide moiety $(176 \mathrm{Da})$. The $\mathrm{MS}^{3}$ spectral spectrum of $m / z 421$ released product ions at $m / z 403,331$, and 301;

\begin{tabular}{|c|c|c|c|c|c|c|c|c|}
\hline \multirow[b]{2}{*}{ Compound } & \multirow[b]{2}{*}{$\begin{array}{l}\text { Spiked Conc. } \\
\text { (ng/ml) }\end{array}$} & \multicolumn{3}{|c|}{ Intra-day } & \multicolumn{3}{|c|}{ Inter-day } & \multirow{2}{*}{$\begin{array}{c}\text { Recovery } \\
\text { (\%) }\end{array}$} \\
\hline & & Mean \pm SD & Accuracy (\%) & $\begin{array}{c}\text { RSD } \\
(\%)\end{array}$ & Mean \pm SD & $\begin{array}{c}\text { Accuracy } \\
(\%)\end{array}$ & $\begin{array}{c}\text { RSD } \\
(\%)\end{array}$ & \\
\hline \multirow{3}{*}{ neomangiferin } & 300 & $302.08 \pm 13.90$ & 100.7 & 4.6 & $300.85 \pm 27.79$ & 100.3 & 9.2 & $56.6 \pm 2.3$ \\
\hline & 6000 & $5640.45 \pm 238.99$ & 94.0 & 4.2 & $6067.74 \pm 649.18$ & 101.1 & 10.7 & $61.2 \pm 3.2$ \\
\hline & 12000 & $11694.2 \pm 151.65$ & 97.5 & 1.3 & $12219.85 \pm 1032.6$ & 101.8 & 8.4 & $59.8 \pm 1.9$ \\
\hline \multirow{3}{*}{ mangiferin } & 100 & $100.15 \pm 2.12$ & 100.2 & 2.1 & $105.55 \pm 6.54$ & 105.5 & 6.2 & $77.8 \pm 5.1$ \\
\hline & 600 & $583.36 \pm 13.38$ & 97.2 & 2.3 & $577.64 \pm 7.41$ & 96.3 & 1.3 & $81.5 \pm 1.7$ \\
\hline & 1200 & $1213.36 \pm 57.14$ & 101.1 & 4.7 & $1195.51 \pm 20.64$ & 99.7 & 1.7 & $75.0 \pm 2.5$ \\
\hline
\end{tabular}

Table 1: Intra- and inter-day precision, accuracy and recovery for determination of neomangiferin and mangiferin in plasma $(n=5)$.

\begin{tabular}{|c|c|c|c|c|c|c|}
\hline \multirow{2}{*}{ Condition } & \multicolumn{3}{|c|}{ Neomangiferin (Stability (\%) \pm SD) } & \multicolumn{3}{|c|}{ Mangiferin (Stability (\%) \pm SD) } \\
\hline & 300 (ng/ml) & $6000(\mathrm{ng} / \mathrm{ml})$ & $12000(\mathrm{ng} / \mathrm{ml})$ & $100(\mathrm{ng} / \mathrm{ml})$ & $600(\mathrm{ng} / \mathrm{ml})$ & $1200(\mathrm{ng} / \mathrm{ml})$ \\
\hline Short term stability & $101.46 \pm 0.43$ & $103.03 \pm 3.10$ & $100.27 \pm 0.91$ & $99.61 \pm 8.11$ & $100.16 \pm 2.49$ & $93.91 \pm 1.11$ \\
\hline Freeze-thaw stability & $96.94 \pm 6.41$ & $99.15 \pm 4.64$ & $98.07 \pm 5.82$ & $95.51 \pm 9.85$ & $104.03 \pm 7.27$ & $100.89 \pm 1.19$ \\
\hline Long term stability & $98.64 \pm 1.94$ & $101.86 \pm 2.47$ & $101.31 \pm 7.69$ & $100.16 \pm 6.16$ & $95.61 \pm 2.15$ & $103.06 \pm 3.3$ \\
\hline
\end{tabular}

Table 2: Assessment of stability of neomangiferin and mangiferin in plasma $(n=3)$. 
A

Neomangiferin

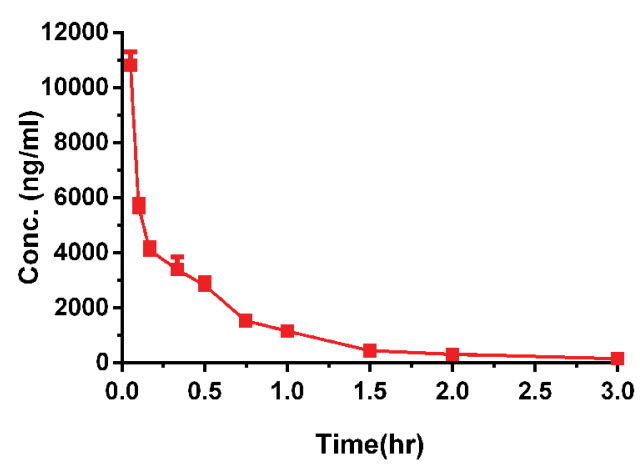

C

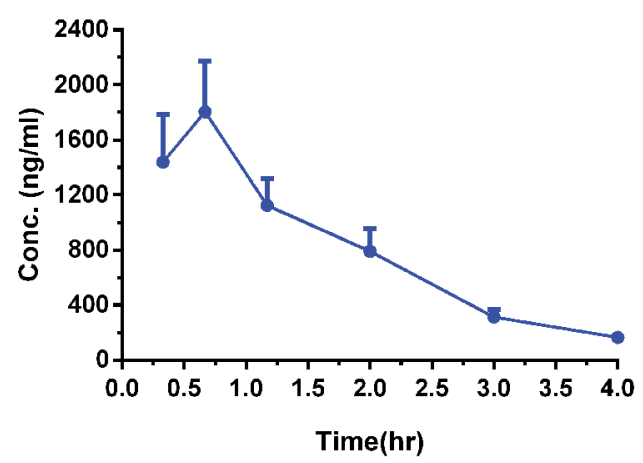

B Mangiferin

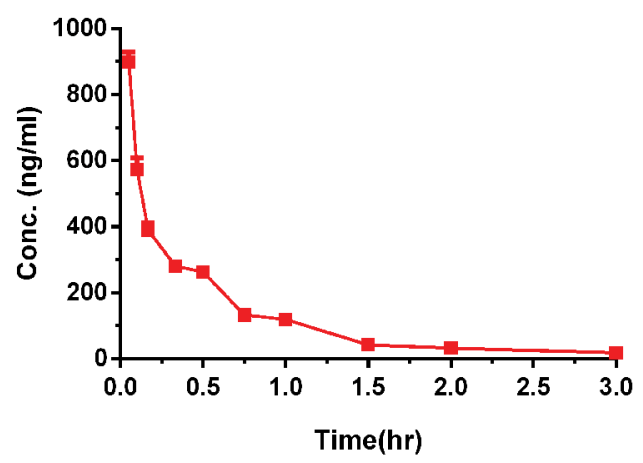

D Mangiferin

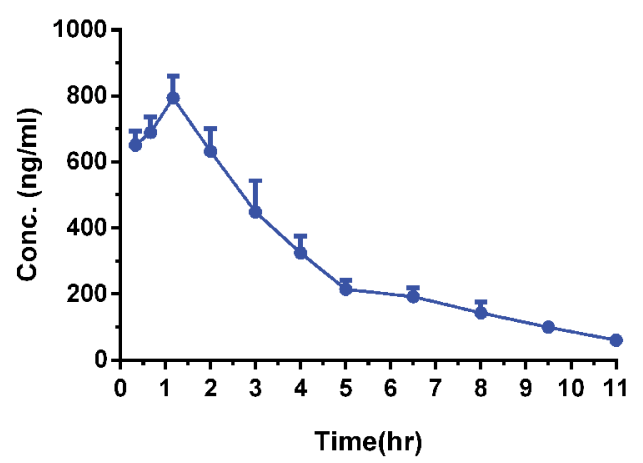

Figure 3: Mean plasma concentration-time profiles of (A) neomangiferin after neomangiferin was intravenously injected to rats, (B) mangiferin after neomangiferin was intravenously injected to rats, $(C)$ neomangiferin after neomangiferin was orally administered to rats, and (D) mangiferin after neomangiferin was orally administered to rats (each point represents mean $\pm \mathrm{SE}, n=5$ ).

\begin{tabular}{|c|c|c|c|c|}
\hline \multirow{2}{*}{ Parameter } & \multicolumn{2}{|c|}{ Healthy rats (i.v.) } & \multicolumn{2}{|c|}{ Healthy rats (p.o.) } \\
\hline & neomangiferin & mangiferin & neomangiferin & mangiferin \\
\hline $\mathrm{C}_{\max }(\mathrm{ng} / \mathrm{mL})$ & $10817.9 \pm 1089.7$ & $899.48 \pm 64.71$ & $1804.28 \pm 818.15$ & $824.48 \pm 115.74$ \\
\hline $\mathrm{T}_{\max }(\mathrm{h})$ & 0.05 & 0.05 & 0.67 & $1.54 \pm 0.82$ \\
\hline$t_{1 / 2}(h)$ & $0.95 \pm 0.06$ & $0.73 \pm 0.15$ & $0.95 \pm 0.31$ & $3.22 \pm 1.07$ \\
\hline $\mathrm{AUC}_{0-\mathrm{t}}(\mathrm{ng} \mathrm{h} / \mathrm{mL})$ & $4372.03 \pm 658.52$ & $392.78 \pm 54.98$ & $3107.96 \pm 1117.93$ & $3276.08 \pm 500.96$ \\
\hline $\mathrm{AUC}_{0-\infty}(\mathrm{ng} \mathrm{h} / \mathrm{mL})$ & $4578.82 \pm 680.4$ & $411.52 \pm 64.66$ & $3333.86 \pm 1147.37$ & $3758.04 \pm 448.14$ \\
\hline $\mathrm{MRT}_{0-\mathrm{t}}(\mathrm{h})$ & $0.54 \pm 0.01$ & $0.59 \pm 0.06$ & $1.38 \pm 0.13$ & $3.27 \pm 0.16$ \\
\hline $\mathrm{MRT}_{0-\infty}(\mathrm{h})$ & $0.72 \pm 0.02$ & $0.74 \pm 0.13$ & $1.69 \pm 0.19$ & $4.79 \pm 1.57$ \\
\hline CL/CL_F (L/kg/h) & $0.4 \pm 0.06$ & $4.46 \pm 0.69$ & $132.43 \pm 46.6)$ & $107.73 \pm 13.57$ \\
\hline
\end{tabular}

Table 3: Pharmacokinetic parameters of the neomangiferin and mangiferin in rats after oral administration (p.o.) or intravenous administration (i.v.) of neomangiferin (mean $\pm \mathrm{SD}, \mathrm{n}=5)$.

these ions were characteristic of mangiferin. Generally, in reversed phase HPLC, the more sugar moieties attached, the earlier the retention time. The earlier retention times of M1 and M2 are in keeping with them being mangiferin conjugated with one glucuronide. Therefore, the two metabolites were tentatively assigned as isomers of mangiferin glucuronide.

Metabolites M3 and M4 were detected at 12.8 and $13.5 \mathrm{~min}$, respectively. The two metabolites afforded an $[\mathrm{M}-\mathrm{H}]^{-}$ion at $\mathrm{m} / z 611$, which was $14 \mathrm{Da}$ higher than those of M1 and M2. The characteristic
MS 2 product ions at $m / z 593,521,491,435,345$, and 315 all increased by $14 \mathrm{Da}$ compared with those of M1 and M2. This observation suggests the addition of a methyl group to the mangiferin glucuronide moiety. The addition of a methyl moiety to mangiferin glucuronide markedly increases reverse phase HPLC retentions. Thus, the two metabolites were identified as isomers of monomethyl mangiferin glucuronide.

The retention times of M6, M10, and M12 were 17.5, 19.9, and 20.9 min, respectively. The metabolites showed similar $[\mathrm{M}-\mathrm{H}]^{-}$ions at $\mathrm{m} / \mathrm{z}$ 435 , which was $14 \mathrm{Da}$ higher than that of mangiferin and corresponded 


\begin{tabular}{|c|c|c|c|c|c|c|c|c|c|c|c|c|c|}
\hline \multirow{2}{*}{ No } & \multirow{2}{*}{ Identification } & \multirow{2}{*}{$\underset{(\min )}{R_{t}}$} & \multicolumn{9}{|c|}{ Location } & \multirow{2}{*}{$\begin{array}{l}\text { Precursor ion } \\
(\mathrm{m} / \mathrm{z})\end{array}$} & \multirow{2}{*}{$\begin{array}{l}\text { Data-dependent } \mathrm{MS}^{\mathrm{n}} \text { data(\% base peak) } \\
\qquad(\mathrm{m} / \mathrm{z})\end{array}$} \\
\hline & & & 1 & 2 & 3 & 4 & 5 & 6 & 7 & 8 & 9 & & \\
\hline M0 & neomangiferin & 11.4 & + & + & - & + & + & - & + & + & - & $583[\mathrm{M}-\mathrm{H}]^{-}$ & $\begin{array}{c}\mathrm{MS}^{2}[583]: \text { 565(28), } 493(100), 463(61), 421(12), 331(9), \\
301(7) \\
M^{3}[421]: 403(19), 331(65), 301(100)\end{array}$ \\
\hline M1 & mangiferin glucuronide & 11.2 & - & - & - & - & - & - & - & + & - & $597[\mathrm{M}-\mathrm{H}]^{-}$ & $\begin{array}{c}\mathrm{MS}^{2}[597]: \text { 421(100), 331(6), 301(11) } \\
\mathrm{MS}^{3}[421]: \text { 403(12), 331(73), 301(100), 259(9) }\end{array}$ \\
\hline M2 & mangiferin glucuronide & 12.2 & - & - & + & - & + & - & - & + & - & $597[\mathrm{M}-\mathrm{H}]^{-}$ & $\begin{array}{c}\mathrm{MS}^{2}[597]: 579(39), 507(60), 477(65), 421(100), 331(79), \\
301(55) \\
M^{3}[421]: 403(15), 331(100), 301(80)\end{array}$ \\
\hline M3 & $\begin{array}{l}\text { monomethyl mangiferin } \\
\text { glucuronide }\end{array}$ & 12.8 & - & - & - & - & + & - & - & + & - & $611[\mathrm{M}-\mathrm{H}]^{-}$ & $\begin{array}{c}\mathrm{MS}^{2}[611]: 593(7), 521(3), 491(12), 435(100), 345(5) \\
315(25), 272(3) \\
\mathrm{MS}^{3}[435]: 417(4), 345(43), 315(100), 272(11)\end{array}$ \\
\hline M4 & $\begin{array}{l}\text { monomethyl mangiferin } \\
\text { glucuronide }\end{array}$ & 13.5 & - & - & - & - & + & - & - & + & - & $611[\mathrm{M}-\mathrm{H}]^{-}$ & $\begin{array}{c}\mathrm{MS}^{2}[611]: 435(100), 345(7), 315(9) \\
\mathrm{MS}^{3}[435]: 417(12), 345(59), 315(100), 273(3)\end{array}$ \\
\hline M5 & mangiferin & 16.1 & + & - & + & + & + & + & + & + & + & $421[\mathrm{M}-\mathrm{H}]^{-}$ & $\begin{array}{c}\mathrm{MS}^{2}[421]: \text { 403(17), 331(73), 301(100) } \\
\mathrm{MS}^{3}[331]: \text { 259(93) }\end{array}$ \\
\hline M6 & monomethyl mangiferin & 17.5 & - & - & + & - & - & + & - & - & - & $435[\mathrm{M}-\mathrm{H}]^{-}$ & $\begin{array}{c}\mathrm{MS}^{2}[435]: \text { 345(3), 315(100), 287(44), 272(28) } \\
\mathrm{MS}^{3}[315]: 259(100), 272(1)\end{array}$ \\
\hline M7 & norathyriol diglucuronide & 17.8 & - & - & - & - & + & - & - & - & - & $611[\mathrm{M}-\mathrm{H}]^{-}$ & $\begin{array}{c}\mathrm{MS}^{2}[611]: 435(100), 259(12) \\
\mathrm{MS}^{3}[435]: 259(100)\end{array}$ \\
\hline M8 & $\begin{array}{l}\text { norathyriol glycoside } \\
\text { glucuronide }\end{array}$ & 18.3 & - & - & - & - & + & - & - & - & - & $597[\mathrm{M}-\mathrm{H}]^{-}$ & $\begin{array}{c}\mathrm{MS}^{2}[597]: \text { 435(100), 417(61), 259(23) } \\
\mathrm{MS}^{3}[435]: 259(100)\end{array}$ \\
\hline M9 & $\begin{array}{l}\text { norathyriol glycoside } \\
\text { glucuronide }\end{array}$ & 19.0 & - & - & - & - & + & - & - & - & - & $597[\mathrm{M}-\mathrm{H}]^{-}$ & $\begin{array}{c}\mathrm{MS}^{2}[597]: \text { 421(100), 259(4) } \\
\mathrm{MS}^{3}[421]: 259(100)\end{array}$ \\
\hline M10 & monomethyl mangiferin & 19.9 & - & - & + & - & + & - & - & - & + & $435[\mathrm{M}-\mathrm{H}]^{-}$ & $\begin{array}{c}\mathrm{MS}^{2}[435]: 417(4), 345(12), 315(100), 272(14), 259(18) \\
\mathrm{MS}^{3}[315]: 315(100), 272(6)\end{array}$ \\
\hline M11 & norathyriol diglucuronide & 20.2 & - & - & - & - & + & - & - & - & - & $611[\mathrm{M}-\mathrm{H}]^{-}$ & $\begin{array}{c}\mathrm{MS}^{2}[611]: \text { 435(100), 258(5) } \\
\mathrm{MS}^{3}[435]: 259(100)\end{array}$ \\
\hline M12 & monomethyl mangiferin & 20.9 & - & + & + & + & + & + & - & - & + & $435[\mathrm{M}-\mathrm{H}]^{-}$ & $\begin{array}{c}\mathrm{MS}^{2}[435]: \text { 417(5), 345(30), 315(100), 272(8) } \\
\mathrm{MS}^{3}[315]: 272(3)\end{array}$ \\
\hline M13 & $\begin{array}{l}\text { monomethyl norathyriol } \\
\text { diglucuronide }\end{array}$ & 21.3 & - & - & - & - & + & - & - & - & - & $625[\mathrm{M}-\mathrm{H}]^{-}$ & $\begin{array}{l}\mathrm{MS}^{2}[625]: 449(100), 273(50) \\
\mathrm{MS}^{3}[449]: 273(100) \\
\operatorname{MS}^{4}[273]: 258(4)\end{array}$ \\
\hline M14 & mangiferin sulfate & 22.1 & - & - & - & - & - & - & - & - & + & $501[\mathrm{M}-\mathrm{H}]^{-}$ & $\begin{array}{c}\mathrm{MS}^{2}[501] \text { : 421(100), 301(4) } \\
\mathrm{MS}^{3}[421]: \text { 403(12), 331(93), 301(100), 259(7) }\end{array}$ \\
\hline M15 & norathyriol glycoside & 25.5 & - & - & - & - & + & - & - & - & - & $421[\mathrm{M}-\mathrm{H}]-$ & MS2 [421]: 259(100) \\
\hline M16 & dimethyl mangiferin & 25.6 & - & - & - & - & + & - & - & - & - & $449[\mathrm{M}-\mathrm{H}]-$ & $\begin{array}{c}\text { MS2 [449]: } 345(5), 315(18), 273(100) \\
\text { MS3 [273]: 258(16) }\end{array}$ \\
\hline M17 & $\begin{array}{l}\text { monodehydroxyl } \\
\text { norathyriol glucuronide }\end{array}$ & 25.7 & - & - & - & - & + & - & - & - & - & $419[\mathrm{M}-\mathrm{H}]^{-}$ & $\mathrm{MS}^{2}[419]: 243(100)$ \\
\hline M18 & dimethyl mangiferin & 26.2 & - & - & - & - & + & - & - & - & - & $449[\mathrm{M}-\mathrm{H}]^{-}$ & $\begin{array}{l}\mathrm{MS}^{2}[449]: 315(4), 273(100) \\
\mathrm{MS}^{3}[273]: 258(23)\end{array}$ \\
\hline M19 & norathyriol glucuronide & 27.5 & - & - & - & + & + & - & - & - & - & $435[\mathrm{M}-\mathrm{H}]^{-}$ & $\mathrm{MS}^{2}[435]: 259(100)$ \\
\hline M20 & $\begin{array}{l}\text { monodehydroxyl } \\
\text { norathyriol glucuronide }\end{array}$ & 27.9 & - & - & - & - & + & - & - & - & - & $419[\mathrm{M}-\mathrm{H}]^{-}$ & $\mathrm{MS}^{2}$ [419]: 243(100) \\
\hline M21 & $\begin{array}{l}\text { monomethyl mangiferin } \\
\text { sulfate }\end{array}$ & 28.0 & - & - & + & + & - & - & + & + & + & $515[\mathrm{M}-\mathrm{H}]^{-}$ & $\begin{array}{c}\mathrm{MS}^{2}[515]: \text { 435(100), 345(5), 315(5) } \\
\mathrm{MS}^{3}[435]: 417(19), 345(59), 315(100)\end{array}$ \\
\hline M22 & $\begin{array}{l}\text { monomethyl norathyriol } \\
\text { glucuronide }\end{array}$ & 28.2 & - & - & - & - & + & - & - & - & - & $449[\mathrm{M}-\mathrm{H}]^{-}$ & $\begin{array}{c}\mathrm{MS}^{2}[449]: 273(100), 258(30) \\
\mathrm{MS}^{3}[273]: 258(52)\end{array}$ \\
\hline M23 & norathyriol glucuronide & 28.6 & - & - & - & + & + & - & - & - & - & $435[\mathrm{M}-\mathrm{H}]^{-}$ & $\mathrm{MS}^{2}[435]: 259(100)$ \\
\hline M24 & $\begin{array}{l}\text { monodehydroxyl } \\
\text { norathyriol glucuronide }\end{array}$ & 28.7 & - & - & - & - & + & - & - & - & - & $419[\mathrm{M}-\mathrm{H}]^{-}$ & $\begin{array}{l}\operatorname{MS}^{2}[419]: 243(100) \\
\operatorname{MS}^{3}[243]: 199(3)\end{array}$ \\
\hline M25 & $\begin{array}{l}\text { monomethyl norathyriol } \\
\text { glucuronide }\end{array}$ & 30.6 & - & - & - & + & + & - & - & - & - & $449[\mathrm{M}-\mathrm{H}]^{-}$ & $\begin{array}{l}\mathrm{MS}^{2}[449]: 273(100) \\
\mathrm{MS}^{3}[273]: 258(19)\end{array}$ \\
\hline M26 & $\begin{array}{l}\text { didehydroxyl norathyriol } \\
\text { glucuronide }\end{array}$ & 32.2 & - & - & - & - & + & - & - & - & - & $403[\mathrm{M}-\mathrm{H}]^{-}$ & $\mathrm{MS}^{2}$ [403]: 227(100) \\
\hline M27 & $\begin{array}{l}\text { monomethyl norathyriol } \\
\text { glucuronide }\end{array}$ & 33.1 & - & - & - & + & + & - & - & - & - & $449[\mathrm{M}-\mathrm{H}]^{-}$ & $\begin{array}{l}\mathrm{MS}^{2}[449]: 273(100) \\
\mathrm{MS}^{3}[273]: 258(10) \\
\mathrm{MS}^{3}[258]: 231(9)\end{array}$ \\
\hline M28 & norathyriol & 37.4 & - & - & - & - & + & + & - & - & - & $519[2 \mathrm{M}-\mathrm{H}]^{-}$ & $\begin{array}{l}\mathrm{MS}^{2}[519]: 259(100) \\
\mathrm{MS}^{3}[259]: 231(1)\end{array}$ \\
\hline M29 & $\begin{array}{l}\text { norathyriol glycoside } \\
\text { sulfate }\end{array}$ & 38.1 & - & - & - & - & + & - & - & - & - & $501[\mathrm{M}-\mathrm{H}]^{-}$ & $\begin{array}{c}\mathrm{MS}^{2}[501]: \text { : } 421(100), 259(100) \\
\mathrm{MS}^{3}[421]: 258(100)\end{array}$ \\
\hline M30 & monomethyl norathyriol & 43.8 & - & - & - & - & + & - & - & - & - & $273[\mathrm{M}-\mathrm{H}]^{-}$ & $\begin{array}{c}\mathrm{MS}^{2}[273]: 258(100) \\
\mathrm{MS}^{3}[258]: 230(1)\end{array}$ \\
\hline M31 & $\begin{array}{l}\text { monodehydroxyl } \\
\text { norathyriol }\end{array}$ & 44.9 & - & - & - & - & + & + & - & - & - & $243[\mathrm{M}-\mathrm{H}]^{-}$ & $\mathrm{MS}^{2}[243]: 199(100)$ \\
\hline
\end{tabular}




\begin{tabular}{|l|l|l|l|l|l|l|l|l|l|l|l|l|l|}
\hline M32 & monomethyl norathyriol & 46.4 & - & - & - & - & + & + & - & - & - & $273[\mathrm{M}-\mathrm{H}]^{-}$ & $\begin{array}{c}\mathrm{MS}^{2}[273]: 258(100) \\
\mathrm{MS}^{3}[258]: 230(9)\end{array}$ \\
\hline M33 & monomethyl norathyriol & 51.9 & - & - & - & - & - & + & - & - & - & $273[\mathrm{M}-\mathrm{H}]$ & $\mathrm{MS}^{2}[273]: 258(100)$ \\
\hline
\end{tabular}
1 3, plasma, o 48h urine, o 48h feces after intravenous injection of neomangiferin to healhy rats, respectively; 4 6, plasma, o 48h urine, o 48h feces after oral
administration of neomangiferin to healthy rats, respectively; 7 9, plasma, o 48h urine, o 48h feces after oral administration of neomangiferin to germ-free rats, respectively;

Table 4: Retention time, identification, detection, and $\mathrm{MS}^{\mathrm{n}}$ data of neomangiferin and its metabolites.

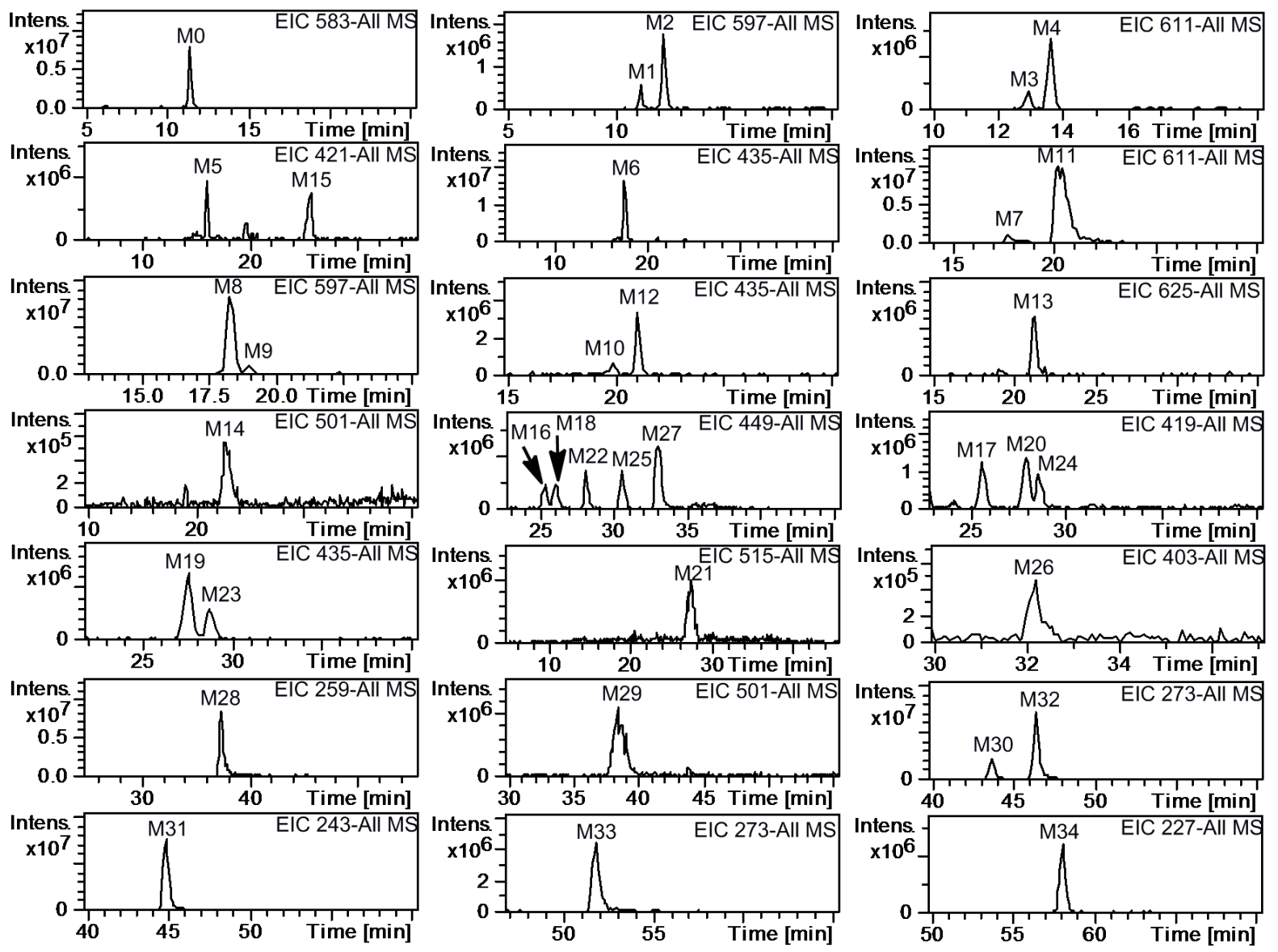

Figure 4: Extracted ion chromatograms (EICs) of neomangiferin and its metabolites. Intens., intensity.

to the methyl conjugate of mangiferin. The MS ${ }^{2}$ fragmentation ions at $\mathrm{m} / \mathrm{z} 345,315,287$, and 272 all increased by 14 Da compared with those of mangiferin. Because of the methyl attached, they eluted at a much later point than that of mangiferin. These results indicate that M6, M10, and M12 were isomers of monomethyl mangiferin.

M7 and M11, eluted at 17.8 and $20.2 \mathrm{~min}$, respectively, each showed a $[\mathrm{M}-\mathrm{H}]^{-}$ion at $\mathrm{m} / z$ 611. The $\mathrm{MS}^{2}$ fragmentation displayed characteristic fragment ions at $\mathrm{m} / z 435$ and 259 derived from the successive loss of two glucuronide moieties. Together with the results of previous studies [17], we unambiguously identified M7 and M11 as the isomers of norathyriol diglucuronide.

M8 and M9, eluted at the retention time of 18.3 and $19.0 \mathrm{~min}$, were detected with a $[\mathrm{M}-\mathrm{H}]^{-}$ion at $m / z$ 597. The $\mathrm{MS}^{2}$ spectrum of M8 displayed typical fragment ions at $m / z 435$ and 259 , which resulted from the stepwise loss of a hexose and a glucuronide unit. The $\mathrm{MS}^{2}$ fragmentation of M9 was similar to that of M8, suggesting that M8 and M9 were isomers. Thus, M8 and M9 were tentatively identified as the isomers of norathyriol glycoside glucuronide.
M13, eluted at 21.3 min retention time, was detected with a $[\mathrm{M}-\mathrm{H}]^{-}$ion at $\mathrm{m} / z$ 625. The $\mathrm{MS}^{2}, \mathrm{MS}^{3}$, and $\mathrm{MS}^{4}$ fragmentations showed characteristic fragment ions at $m / z 449,273$, and 258 derived from the successive loss of two glucuronide and one monomethyl group. Therefore, the M13 was tentatively identified as monomethyl norathyriol diglucuronide.

M14, eluted at $22.1 \mathrm{~min}$, displayed a $[\mathrm{M}-\mathrm{H}]^{-}$ion at $\mathrm{m} / z 501$, which was $80 \mathrm{Da}$ higher than that of mangiferin. The MS ${ }^{2}$ fragmentation revealed a fragment ion at $m / z 421$, which was formed by the loss of a sulfate group. The MS/MS spectrum of $m / z 401$ released product ions at $m / z 403,331$, 301 , and 259, which were the characteristic ions of mangiferin. Therefore, the M14 was tentatively identified as mangiferin sulfate.

M15, present at $25.5 \mathrm{~min}$, showed a $[\mathrm{M}-\mathrm{H}]^{-}$ion at $m / z 421$, which was $162 \mathrm{Da}$ higher than that of norathyriol. The $\mathrm{MS}^{2}$ fragmentation showed a fragment ion at $m / z 259$ derived from the loss of a glycoside unit. Comparison with norathyriol, M15 showed lower retention time. Therefore, the M15 was tentatively identified as norathyriol glycoside. 
M16 and M18, eluted at retention times 25.6 and $26.2 \mathrm{~min}$ showed a $[\mathrm{M}-\mathrm{H}]^{-}$ion at $\mathrm{m} / z 449$, which was $28 \mathrm{Da}$ higher than that of mangiferin. The $\mathrm{MS}^{2}$ fragmentation ions at $\mathrm{m} / \mathrm{z} 345,315$, and 273 all increased by $28 \mathrm{Da}$ compared with those of mangiferin. The MS/ MS spectrum at $m / z 273$ provided a product ion at $m / z 403$, which was formed by the loss of a dimethyl group. Due to the dimethyl conjugated, M16 and M18 showed higher retention time than the monomethyl mangiferin. Therefore, the two metabolites were tentatively identified as isomers of dimethyl mangiferin.

M19 and M23, eluted at retention times 27.5 and $28.6 \mathrm{~min}$, showed a $[\mathrm{M}-\mathrm{H}]^{-}$ion at $m / z 435$, which was $176 \mathrm{Da}$ higher than that of norathyriol. The fragment ion at $m / z 259$ was formed by the loss of a glucuronide group. Therefore, the two metabolites were tentatively identified as isomers of norathyriol glucuronide.

M17, M20, and M24, eluted at retention times 25.7, 27.9, and 28.7 min, showed a $[\mathrm{M}-\mathrm{H}]^{-}$ion at $m / z 419$. The fragment ion at $m / z 243$ was formed by the loss of a glucuronide group. Previous experimental results confirmed the existence of monodehydroxylated and didehydroxylated metabolites of norathyriol in rat urine sample after oral administration of mangiferin [17]. Therefore, M17, M20, and M24 were tentatively identified as isomers of monodehydroxyl norathyriol glucuronide.

$\mathrm{M} 21$, eluted at 28.0 min retention time, was detected with a $[\mathrm{M}-\mathrm{H}]^{-}$ ion at $m / z 515$. The fragment ion at $m / z 435$ was formed by the loss of a sulfate group. The MS ${ }^{3}$ product ions at $m / z 417,345$, and 315 were the same as those of M6, M10, or M12, indicating that M21 was the further sulfated conjugate of M6, M10, or M12. M21 showed higher retention time than mangiferin sulfate. Therefore, M21 was tentatively identified as monomethyl mangiferin sulfate.

M22, M25, and M27, eluted at retention times 28.2, 30.6, and 33.1 min, similarly showed a $[\mathrm{M}-\mathrm{H}]^{-}$ion at $m / z 449$, which was $14 \mathrm{Da}$ higher than that of M19 or M23. The MS 2 fragmentation ions at $m / z 273$ was increased by 14 Da compared with those of M19 or M23, suggesting that the metabolites were the further methylated forms of M19 or M23. Therefore, M22, M25, and M27 were tentatively identified as isomers of monomethyl norathyriol glucuronide.

M26, eluted at $32.2 \mathrm{~min}$, showed a $[\mathrm{M}-\mathrm{H}]^{-}$ion at $\mathrm{m} / \mathrm{z} 403$, which was 32 Da lower than that of M19 or M23, and corresponded to M19 or M23 with loss of two hydroxyl groups. The MS ${ }^{2}$ fragmentation showed fragment ions at $m / z 227$ because of the loss of a glucuronide group. The subtraction of hydroxyl groups to a norathyriol glucuronide moiety can cause it to elute later. Therefore, the metabolite was tentatively identified as didehydroxyl norathyriol glucuronide.

M26, eluted at retention time $38.1 \mathrm{~min}$, showed a $[\mathrm{M}-\mathrm{H}]^{-}$ion at $\mathrm{m} / \mathrm{z}$ 501 , which was $80 \mathrm{Da}$ higher than that of M15. The MS ${ }^{2}$ fragmentation showed a fragment ion at $m / z 421$ because of the loss of a sulfate group. Therefore, the metabolite was tentatively identified as norathyriol glycoside sulfate.

M30, M32, and M33, eluted at retention times 43.8, 46.4, and $51.9 \mathrm{~min}$, showed similar $[\mathrm{M}-\mathrm{H}]^{-}$ions at $\mathrm{m} / z 273$, which was $14 \mathrm{Da}$ higher than that of norathyriol. The $\mathrm{MS}^{2}$ product ions at 258 , was consistent with the ion observed in the MS ${ }^{3}$ spectra of M22, M25, or M27. Therefore, the metabolites were tentatively identified as isomers of monomethyl norathyriol.

M31, eluted at retention time $44.9 \mathrm{~min}$, showed a $[\mathrm{M}-\mathrm{H}]^{-}$ion at $\mathrm{m} / \mathrm{z}$ 243 , which was $16 \mathrm{Da}$ lower than that of norathyriol. This observation revealed that M31 was a dehydroxyl product of norathyriol. And M31 have a longer retention time than the norathyriol. Therefore, the metabolite was tentatively identified as monodehydroxyl norathyriol.

\section{Metabolism of neomangiferin in conventional rats}

The proposed metabolic pathways of neomangiferin in conventional and pseudo-germ-free rats after its oral and intravenous injection administration are summarized in Figure 5.

After neomangiferin was intravenously injected to conventional rats, the parent compound could be detected in the plasma and urine. Furthermore, one metabolite (M5) in the plasma, one metabolite (M12) in the urine, and six metabolites (M2, M5, M6, M10, M12, and M21) in the feces were detected. In general, neomangiferin itself was initially deglycosylated to be transformed into mangiferin, which was further subjected to methylation to form the metabolites M6, M10, and M12, glucuronidation to form the metabolite M2 and sulfation to form the metabolite M21, respectively.

After neomangiferin was orally administered to conventional rats, neomangiferin underwent extensive metabolism in vivo to produce more metabolites. The parent compound and 7 metabolites (M5, M12, M19, M21, M23, M25, and M27) in plasma, the parent compound and 29 metabolites (M2-M5, M7-M13, M15-M20, M22-M32, and M34) in the urine, and 8 metabolites (M5, M6, M12, M28, and M31-M34) in the feces were detected. When orally administered to conventional rats, neomangiferin was initially metabolized into mangiferin, and the subsequent metabolic pathways were similar to those in conventional rats after mangiferin was orally administered. Our in vivo metabolic analysis indicated that neomangiferin is metabolized via an intermediate mangiferin to other final metabolites.

After oral administration of neomangiferin to pseudo-germ-free rats, the metabolic pathways of neomangiferin were similar to those in conventional rats after its intravenous injection administration. The parent compound also appeared in the plasma and urine. In addition, 2 metabolites (M5, M21) in the plasma, 6 metabolites (M1-M5, M21) in the urine, and 5 metabolites (M5, M10, M12, M14, M21) in the feces were detected. Deglycosylation, methylation, glucuronidation and sulfation were proposed to be the predominant metabolic pathways of neomangiferin in pseudo-germ-free rat.

\section{Conclusions}

In this study, a simple, accurate, and selective method was established to determine neomangiferin and its metabolite mangiferin in rat plasma simultaneously. The proposed method was applied successfully to conduct a pharmacokinetic analysis of neomangiferin orally and intravenously administered to rats. Pharmacokinetic results revealed that neomangiferin was absorbed and eliminated rapidly in vivo. Neomangiferin was also partly transformed to mangiferin rapidly post-dosing and exhibited poor bioavailability (only $0.32 \%$ ). This poor bioavailability may be partially attributed to the low absorption and extensive metabolism of neomangiferin. Furthermore, 33 metabolites of neomangiferin in rats were identified for the first time. Neomangiferin underwent rapid and extensive phases I and II metabolic processes, such as deglycosylation, dehydroxylation, methylation, glycosylation, glucuronidation, and sulfation, after this component was orally administered to rats. In general, the pharmacokinetic and metabolic profiles, including poor bioavailability, high absorption and elimination rates, easy biotransformation, and some metabolic pathways, of neomangiferin were similar to those of mangiferin. This resemblance may be ascribed to the comparable chemical structures of these compounds. However, these pharmacokinetic characteristics may hinder the development of neomangiferin as a new drug candidate. Our findings could provide critical information to promote further exploration and enhancement of neomangiferin. 

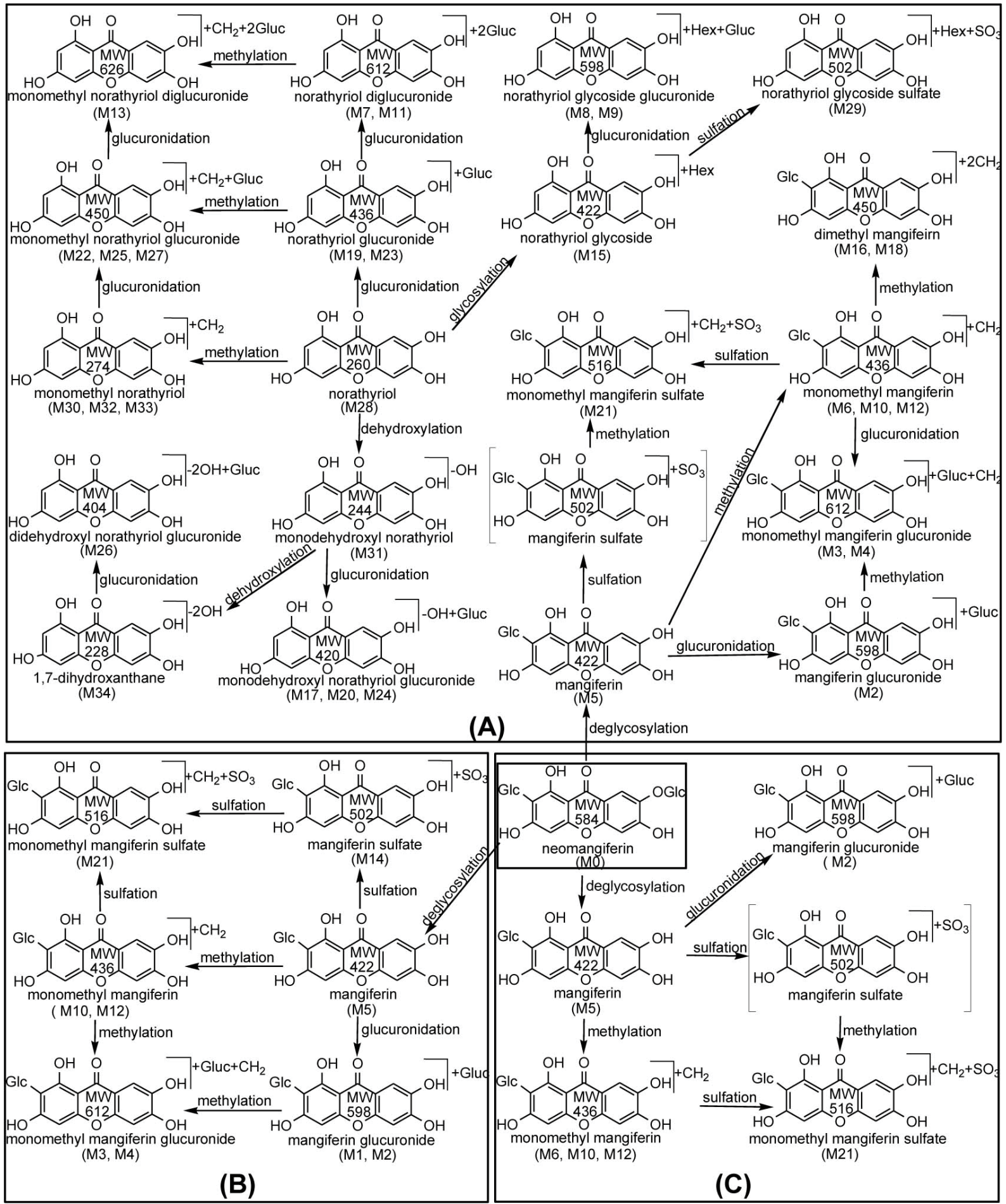

Figure 5: Proposed metabolic pathways of neomangiferin in rats. (A) after oral administration to conventional rats, (B) after oral administration to pseudo-germfree rats; $(C)$ after intravenous injection to conventional rats.

\section{Acknowledgements}

We thank the State Key Program of National Natural Science Foundation of China (Grant No. 81030065) and the National Science and Technology Major Project (Grant Nos. 2009ZX09301-001, 2009ZX09102-121, 2009ZX09501-030, 2012ZX09301001-001) for providing financial support.

\section{References}

1. Hong YF, Han GY, Guo XM (1997) Isolation and structure determination of xanthone glycosides of Anemarrhena asphodeloides. Yao Xue Xue Bao 32 473-475.

2. Miura T, Ichiki H, Iwamoto N, Kato M, Kubo M, et al. (2001) Antidiabetic activity of the rhizoma of Anemarrhena asphodeloides and active components, mangiferin and its glucoside. Biol Pharm Bull 24: 1009-1011.

3. Ichiki H, Miura T, Kubo M, Ishihara E, Komatsu Y, et al. (1998) New antidiabetic compounds, mangiferin and its glucoside. Biol Pharm Bull 21: 1389-1390.

4. Qin L, Han T, Zhang Q, Cao D, Nian H, et al. (2008) Antiosteoporotic chemical 
Citation: Gao Y, Liu H, Hu P, Chen M, Tian X, et al. (2016) The Pharmacokinetics and Metabolism of Neomangiferin, a Major Bioactive Component in Anemarrhena asphodeloides. Med chem (Los Angeles) 6: 385-394. doi:10.4172/2161-0444.1000374

constituents from Er-Xian Decoction, a traditional Chinese herbal formula. J Ethnopharmacol 118: 271-279.

5. Lim SM, Kang GD, Jeong JJ, Choi HS, Kim DH (2016) Neomangiferin modulates the Th17/Treg balance and ameliorates colitis in mice. Phytomedicine 23: 131-140.

6. Ma C, Wang L, Tang Y, Fan M, Xiao H, et al. (2008) Identification of major xanthones and steroidal saponins in rat urine by liquid chromatographyatmospheric pressure chemical ionization mass spectrometry technology following oral administration of Rhizoma Anemarrhenae decoction. Biomed Chromatogr 22: 1066-1083.

7. Cai F, Xu W, Wei H, Sun L, Gao S, et al. (2010) Simultaneous determination of active xanthone glycosides, timosaponins and alkaloids in rat plasma after oral administration of Zi-Shen Pill extract for the pharmacokinetic study by liquid chromatography-tandem mass spectrometry. J Chromatogr B 878: 1845-1854.

8. Das S, Nageshwar Rao B, Satish Rao BS (2011) Mangiferin attenuates methylmercury induced cytotoxicity against IMR-32, human neuroblastoma cells by the inhibition of oxidative stress and free radical scavenging potential. Chem. Biol. Interact 193: 129-140.

9. Duang XY, Wang Q, Zhou XD, Huang DM (2011) Mangiferin: a possible strategy for periodontal disease to therapy. Med Hypotheses 76: 486-488.

10. Girón MD, Sevillano N, Salto R, Haidour A, Manzano M, et al. (2009) Salacia oblonga extract increases glucose transporter 4-mediated glucose uptake in L6 rat myotubes: role of mangiferin. Clin Nutr 28: 565-574.

11. Shoji K, Tsubaki M, Yamazoe Y, Satou T, Itoh T, et al. (2011) Mangiferin induces apoptosis by suppressing $\mathrm{Bcl}-\mathrm{xL}$ and $\mathrm{XIAP}$ expressions and nuclear entry of NF-kB in HL-60 cells. Arch Pharm Res 34: 469-475.

12. Savikin K, Menkovic N, Zdunic G, Stevic T, Radanovic D, et al. (2009) Antimicrobial activity of Gentiana lutea L. extracts. Z Naturforsch C 64: 339-342.
13. Wang RR, Gao YD, Ma CH, Zhang XJ, Huang CG, et al. (2011) Mangiferin an anti-HIV-1 agent targeting protease and effective against resistant strains. Molecules 16: 4264-4277.

14. Jagetia GC, Baliga MS (2005) Radioprotection by mangiferin in DBAxC57BL mice: a preliminary study. Phytomedicine 12: 209-215.

15. He C, Li J, Xu N, Wang R, Li Z, et al. (2015) Pharmacokinetics, bioavailability, and metabolism of Notoginsenoside $\mathrm{Fc}$ in rats by liquid chromatography/ electrospray ionization tandem mass spectrometry. J Pharm Biomed Anal 109 150-157.

16. Wang $\mathrm{H}$, Ye G, Ma CH, Tang $\mathrm{YH}$, Fan MS, et al. (2007) Identification and determination of four metabolites of mangiferin in rat urine. J Pharm Biomed Anal 45: 793-798.

17. Liu H, Wang K, Tang Y, Sun Z, Jian L, et al. (2011) Structure elucidation of in vivo and in vitro metabolites of mangiferin. J Pharm Biomed Anal 55: 1075-1082.

18. Liu H, Wu B, Pan G, He L, Li Z, et al. (2012) Metabolism and pharmacokinetics of mangiferin in conventional rats, pseudo-germ-free rats, and streptozotocininduced diabetic rats. Drug Metab Dispos 40: 2109-2118.

19. Han D, Chen C, Zhang C, Zhang Y, Tang X (2010) Determination of mangiferin in rat plasma by liquid-liquid extraction with UPLC-MS/MS. J Pharm. Biomed. Anal. 51: 260-263.

20. Qiu X, Zhao JL, Hao C, Yuan C, Tian N, et al. (2016) Simultaneous determination of mangiferin and neomangiferin in rat plasma by UPLC-MS/MS and its application for pharmacokinetic study. J Pharm Biomed Anal 124: 138-142.

21. Xie YY, Wang XM, Wang SH, Wang YM, Tian HF, et al. (2015) Metabolism and pharmacokinetics of major polyphenol components in rat plasma after oral administration of total flavonoid tablet from Anemarrhenae Rhizoma. J. Chromatogr. B Analyt. Technol. Biomed. Life Sci 15: 30327-30335. 